\title{
Der Neoricardianismus: eine Fortsetzung klassischer Theorie: Kommentar zum Beitrag von Bertram Schefold
}

\author{
Carl Christian von Weizsäcker
}

Online publiziert: 1. April 2019

(C) List-Gesellschaft e.V. 2019

In meinem Kommentar beschränke ich mich auf zwei Punkte: die Interpretation des Wicksell-Effektes und die Frage der Substitution infolge veränderter Zinssätze. Beide Punkte behandle ich im Rahmen einer Steady-State-Analyse. Ich nehme nicht Stellung zu der „Philosophie“ des Ansatzes von Sraffa, dessen Schule sich ja als dezidiert „anti-neoklassisch“ versteht und sich in der Tradition der „klassischen“ Schule, speziell Ricardos sieht. Sraffas großes Editionswerk einer Gesamtausgabe der Werke und Briefe David Ricardos besitzt meine große Bewunderung.

\section{Der Wicksell-Effekt}

Wie ein roter Faden durchzieht der Wicksell-Effekt, speziell der anti-neoklassische Wicksell-Effekt, Schefolds Darstellung. Von diesem behauptet Schefold, dass er einem instabilen Gleichgewicht entspreche. Für Schefold ist diese Instabilität einer der wesentlichen Kritikpunkte an der neoklassischen Kapitaltheorie.

Unter dem Wicksell-Effekt versteht die Kapitaltheorie folgendes: Man stellt für eine Volkswirtschaft gedanklich unterschiedliche Zustände nebeneinander, die sich durch unterschiedlich hohe Profitraten und entsprechende unterschiedliche Lohnsätze entlang einer Lohn-Zinskurve unterscheiden. Nun kann für eine vorgegebene angewandte Produktionstechnik, also für vorgegebene Kapitalgüter, der Wert dieser selben Kapitalgüter verschieden sein, je nachdem, welche Lohn-Zins-Konstellation

\footnotetext{
This commentary article refers to the article available online at https://doi.org/10.1007/s41025-01900156-1. An author's reply to this commentary article is available online at https://doi.org/10.1007/ s41025-019-00161-4.

C. C. von Weizsäcker $(\bowtie)$

MPI zur Erforschung von Gemeinschaftsgütern, Bonn, Deutschland

E-Mail: ccvwzs@t-online.de
} 
vorherrscht. Eine Verschiebung des Werts des vorgegebenen Stocks an Kapitalgütern bei einer Verschiebung der Profitrate und entsprechenden Verschiebung des Lohnsatzes nennt man den Wicksell-Effekt. Sei für vorgegebene Produktionstechnik $\theta$ der Wert des Kapitalgüterstocks pro Arbeiter gleich $v(r ; \theta)$, wobei $r$ die Profitrate ist. Mit der Profitrate $r$ ändert sich zugleich der Reallohn $w(r ; \theta)$. Nun betrachten wir die partielle Ableitung von $v$ nach $r$. Dann definiert Schefold

$$
\begin{gathered}
\frac{\partial v}{\partial r}<0=\text {,neoklassischer Wicksell-Effekt“; } \\
\frac{\partial v}{\partial r}>0=\text {,nicht-neoklassischer Wicksell-Effekt““ }
\end{gathered}
$$

Von letzterem behauptet Schefold, es liege ein instabiles Gleichgewicht vor. Der Gedanke hierbei ist dieser: Ein höherer Zins und ein damit niedrigerer Lohn sollte gemäß neoklassischer Vorstellung dazu führen, dass mehr an Kapital gespart und dafür mehr an Arbeit eingesetzt wird. Das entspräche sozusagen einem stabilen Gleichgewicht. Wenn aber, so offenbar die Idee, beim nicht-neoklassischen WicksellEffekt mit höherer Profitrate und niedrigerem Lohn der Kapitalwert steigt, so ist diese Idee eines stabilen Gleichgewichts gestört. Offenbar geht es hier um die von der $r-$ Veränderung induzierten Umverteilung, die Rückwirkungen auf die Güternachfrage hat, mit der Folge (beim ,nicht-neoklassischen“ Wicksell-Effekt) einer Abnahme der Nachfrage nach Arbeit trotz gesunkenen Lohns.

Ich halte diese Schlussfolgerung für nicht zulässig. Der Stabilitätsgedanke basiert auf der Vorstellung, dass ein niedrigerer Preis die Nachfrage nach dem Gut erhöht und das Angebot senkt. Aber beim Wicksell-Effekt handelt es sich um eine partielle Ableitung, die die eingesetzten Gütermengen gerade konstant hält. Im Geiste dieses Stabilitätsgedankens müsste man also gerade untersuchen, welche Auswirkungen ein höherer Zins auf das Mengengerüst, sprich auf $\theta$ hat. Man kann die Rückwirkungen der Umverteilung auf die Arbeitsnachfrage nur dann untersuchen, wenn man $\theta$ gerade nicht konstant hält. Das werde ich unten im Rahmen einer ,Steady-StateAnalyse" tun.

Wollte man im Ernst das Stabilitätsproblem untersuchen, müsste man aus dem kapitaltheoretischen Rahmen herausspringen. In diesem Rahmen untersucht man Gütermärkte mit Konkurrenzpreisen, die zugleich die Durchschnittskosten und die Grenzkosten repräsentieren (Konstante Skalenerträge). Faktisch sind jedoch die meisten Märkte, auch Wettbewerbsmärkte, durch Aufschläge auf die Grenzkosten charakterisiert. Ich spreche hier von der Asymmetrie der Märkte zwischen Angebotsund Nachfrageseite (hierauf basiert meine Hayek-Kritik in meinem Artikel Weizsäcker 2005: Hayek hat seine eigene Theorie der Informations-Funktion von Marktpreisen nicht zuende gedacht (Hayek 1945). Hätte er das getan, hätte er verstanden, dass die Angebotsseite fast jeden Marktes aus Wettbewerbsgründen Reservekapazitäten hält. Diese rechtfertigen makro-ökonomisch viele der Schlussfolgerungen von Keynes). Das makroökonomische Stabilitätsproblem kann nur sinnvoll analysiert werden, wenn man diese tiefliegende Marktasymmetrie (sie beruht auf dem die Moderne charakterisierenden Prinzip der Arbeitsteilung, siehe Smith (1776), Satz 1) in die Untersuchung einbezieht. Diese Stabilitätsanalyse muss viel eher den automatischen Stabilisator des Staatshaushalts (bzw. sein mögliches Versagen) betrachten als den anti-neoklassischen Wicksell-Effekt. Die nicht antizipierte Entwertung vor- 
handener Kapitalgüter ist primär ein Effekt geringer Gesamtnachfrage und nicht ein Wicksell-Effekt. Insofern führt die von Schefold erwähnte und betonte Keynes'sche Herauslösung der Investitionsfunktion aus dem neoklassischen General-Equilibrium mit perfekten Zukunftsmärkten auf den richtigen Pfad einer Stabilitätsanalyse. Ich sehe nicht, dass die grundlegenden Arbeiten von Keynes und später Minsky irgendwie dadurch ,,verbessert“ werden können, dass man da einen anti-neoklassischen Wicksell-Effekt einführt.

Ich kehre nunmehr jedoch zum kapitaltheoretischen Rahmen zurück. Hier mache ich eine Steady-State-Analyse. Sie steht in der Tradition Eugen von Böhm-Bawerks (vgl. Böhm-Bawerk 1889) (hier eine Seitenbemerkung nicht zu den Neo-Ricardianern, sondern zu den Neo-Austrians: Leider haben diese ihren Gründungsvater Böhm-Bawerk schlecht verstanden. Das fängt schon mit der abenteuerlichen Idee von Ludwig von Mises über den „Urzins“ an, der schon aus entscheidungslogischen Gründen positiv sein müsse. Aber auch Hayeks Kapitaltheorie oder Oskar Morgensterns Kritik am Begriff der durchschnittlichen Produktionsperiode führen weg vom richtigen Kern der Böhm-Bawerkschen Kapitaltheorie. Erst durch das Werk von John Hicks (1939) ist Böhm-Bawerk in die richtige Richtung weiter entwickelt worden. Aber Hicks wird von den Neo-Austrians ignoriert - und erst recht meine Weiterentwicklung in Weizsäcker 1971).

Der Wicksell-Effekt signalisiert nach meiner Auffassung etwas ganz anderes als das, was Schefold ihm zuschreibt. Im Steady State gilt folgende Gleichung

$$
c(g ; \theta)+g v(r ; \theta)=w(r ; \theta)+r v(r ; \theta)
$$

Dabei ist $g$ die Wachstumsrate des Systems, $c$ der volkswirtschaftliche Konsum pro Arbeiter. Die anderen Variablen wurden oben schon definiert. In der Sprache der volkswirtschaftlichen Gesamtrechnung ist die linke Seite der Gleichung die „Verwendungsseite“ des Nettosozialprodukts. Die rechte Seite gibt die Verteilung des Sozialprodukts zwischen Arbeit und Kapital an. Zur vereinfachten Darstellung wähle ich den repräsentativen Warenkorb von Konsumgütern als eine feststehende Maßeinheit, in der die Strömungsgrößen $c$ und $w$ und die Bestandsgröße $v$ gemessen werden. Indessen ist meine hier angedeutete Theorie sehr viel allgemeiner (sie wird in einigen Monaten in einem längeren kapitaltheoretischen Manuskript vorliegen). Zuerst halte ich die physische Zusammensetzung des Kapitalstocks $\theta$ konstant, ebenso die Wachstumsrate des Systems $g$. Für $r \neq g$ können wir schreiben

$$
v(r ; \theta)=\frac{c(g ; \theta)-w(r ; \theta)}{r-g}
$$

Wir können für kapitaltheoretische Modelle davon ausgehen, dass dieser Ausdruck für $v(r ; \theta)$ eine stetig differenzierbare Funktion von $r$ ist. Daher können wir die Regel von L'Hôpital anwenden und erhalten somit bei $r=g$ den Ausdruck

$$
v(r ; \theta)=-w_{1}(r ; \theta)
$$


wobei $w_{1} \equiv \frac{\partial w}{\partial r}$ die partielle Ableitung des Lohnsatzes $w$ nach dem Zinssatz ist. Nun können wir diese partielle Ableitung des Lohnsatzes in der folgenden Form schreiben

$$
w_{1}=-w(r ; \theta) T(r ; \theta)
$$

Dabei hat die Größe $T(r ; \theta)$ die Dimension ,Zeit“. Da der Lohn mit steigendem Zins abnimmt, gilt $T(r ; \theta)>0$. Für die in der Kapitaltheorie verwendeten Modelle hat dieser Zeitausdruck die folgende ökonomische Interpretation: $T(r ; \theta)$ ist die von Hicks (1939) modernisierte durchschnittliche Produktionsperiode, die BöhmBawerk zur Erklärung des Kapitalbedarfs pro Arbeiter verwendet hat. Somit erkennen wir, dass die Böhm-Bawerksche Erklärung des Kapitalbedarfs jedenfalls dann völlig korrekt ist, wenn der Zinssatz gleich der Wachstumsrate ist. Denn es gilt dann

$$
v(r ; \theta)=-w_{1}(r ; \theta)=w(r ; \theta) T(r ; \theta)
$$

Und genau das hat Böhm-Bawerk behauptet: Der Kapitalbedarf pro Arbeiter ist gleich dem Lohnsatz mal durchschnittliche Produktionsperiode.

Die partielle Ableitung von $v$ nach $r$ ist der Wicksell-Effekt. Wir können nun den Wicksell-Effekt für $r \neq g$ berechnen.

$$
\begin{gathered}
v_{1}(r ; \theta) \equiv \frac{\partial v}{\partial r}=\frac{(r-g)\left(-w_{1}(r ; \theta)-c(g ; \theta)\right)+w(r ; \theta)}{(r-g)^{2}} \\
\quad=\frac{-w_{1}(r ; \theta)-v(r ; \theta)}{r-g}=\frac{w(r ; \theta) T(r ; \theta)-v(r ; \theta)}{r-g}
\end{gathered}
$$

Auch hier können wir den Fall $r=g$ wieder mit L'Hôpital lösen

$$
v_{1}(g ; \theta)=w(g ; \theta) \frac{\partial T}{\partial r}+T w_{1}-v_{1}=\frac{w(g ; \theta)}{2}\left\{\frac{\partial T}{\partial r}-T^{2}\right\}
$$

Andererseits können wir die zweite partielle Ableitung des Lohns nach $r$ bilden. Wir erhalten

$$
w_{11} \equiv \frac{\partial w_{1}}{\partial r}=w\left\{T^{2}-\frac{\partial T}{\partial r}\right\}
$$

Für den Fall $r=g$ stellen wir damit fest

Wicksell-Effekt bei $(r=g)=v_{1}(g ; \theta)=-\frac{1}{2} w_{11}(g ; \theta)$

Unterstellen wir jetzt einmal, dass der Wicksell-Effekt entweder für alle Zinssätze „,neoklassisch“ oder für alle Zinssätze ,,anti-neoklassisch“ ist. Der „,neo-klassische“ Wicksell-Effekt bedeutet, dass die Lohn-Zinskurve eine positive zweite Ableitung besitzt, somit konvex verläuft. Der ,anti-neo-klassische“ Wicksell-Effekt bedeutet dann, dass die Lohn-Zinskurve konkav verläuft. 
Wir können dies auch in die Form einer Taylor-Approximation zweiten Grades für die Lohn-Zinskurve gießen, wobei der Ausgangspunkt dieser Taylor-Approximation der Zinssatz $r=g$ ist. Wir erhalten damit

$$
\begin{gathered}
w(r ; \theta) \approx w(g ; \theta)+w_{1}(g ; \theta)(r-g)+\frac{1}{2} w_{11}(g ; \theta)(r-g)^{2} \\
\quad=w(g ; \theta)\{1-(r-g) T(g ; \theta)\}-v_{1}(g: \theta)(r-g)^{2}
\end{gathered}
$$

Der Wicksell-Effekt an der Stelle $r=g$ entspricht damit dem Koeffizienten des quadratischen Terms der Taylor Approximation zweiten Grades für die Lohn-ZinsKurve um den Ausgangspunkt $r=g$.

Es lohnt sich nun, die Formel für den Wicksell-Effekt bzw. die zweite Ableitung der Lohn-Zins-Kurve noch etwas genauer zu betrachten.

Wir haben - wie oben -

$$
w_{11} \equiv \frac{\partial w_{1}}{\partial r}=w\left\{T^{2}-\frac{\partial T}{\partial r}\right\}
$$

Die Schwierigkeit, in die Böhm-Bawerk mit seinen nur sehr rudimentären Mathematik-Kenntnissen geriet, war, dass er die Produktionsperiode $T$ nicht mit Gegenwartswerten berechnete, sondern mit einfachen Arbeitsmengen, die ohne Diskontierung aufgelistet wurden. Hicks verstand, dass man die Produktionsperiode mit Gegenwartswerten berechnen musste. Damit erst wurde dieses Maß abhängig vom verwendeten Zinssatz. Es gibt aber einen (völlig unrealistischen) Spezialfall, bei dem die Produktionsperiode auch dann zinsunabhängig bleibt, wenn man mit Gegenwartswerten rechnet. Das ist das „Point-Input-Point-Output“-Modell. Der typische Produktionsprozess besteht hier darin, dass ein Arbeitsjahr zu einem bestimmten Zeitpunkt $t_{0}$ eingesetzt wird - und daraus zu einem späteren Zeitpunkt $t_{1}$ ein Korb an Konsumgütern entsteht. Die Produktionsperiode $T$ ist dann einfach die Differenz von $t_{1}$ und $t_{0}$ Sie ist dann offenkundig unabhängig von dem Kalkulationszinssatz, mit dem man die Gegenwartswerte berechnet. Dieser völlig unrealistische Spezialfall gibt uns aber einen Hinweis dafür, wo wir die Abhängigkeit der Produktionsperiode vom Zinssatz erklärt bekommen. Es geht um die intertemporale Dispersion der Arbeitsinputs und der Konsumgüteroutputs.

Ich stelle die formale Theorie hierzu nicht da. Ich berichte nur das Ergebnis: Wir betrachten eine im Steady State wachsende (oder mit $g=0$ stationäre) Volkswirtschaft. Wir denken uns diese zerlegt in eine Menge sich überlappender imaginärer „Firmen“, die alle völlig vertikal integriert sind, sodass sie nur Arbeit von außen einkaufen und nur Konsumgüter im Markt verkaufen. Sie verwenden alle die Produktionstechnik $\theta$. Sie unterscheiden sich allein dadurch, dass ihr interner Zeitpunkt Null einem jeweils unterschiedlichen Kalenderzeitpunkt entspricht. Diese imaginären Firmen überlappen sich in einer solchen Weise, dass daraus im Ganzen eben eine Steady State Volkswirtschaft herauskommt (derartige Gedankenexperimente sind in der Geschichte der Kapitaltheorie immer wieder gemacht worden, meist allerdings ist nur der stationäre Fall untersucht worden. Außerdem war man meist mit der angewandten mathematischen Technik etwas unbeholfen). 
Die Produktionsperiode in einem solchen Modell ist dann die Differenz zwischen dem zeitlichen Schwerpunkt der Konsumgüter-Abgabe der imaginären Firma und dem zeitlichen Schwerpunkt der Arbeitsinputs der imaginären Firma. Diese zeitlichen Schwerpunkte werden mit Gegenwartswerten berechnet. Diese so definierte Produktionsperiode ist für alle sich überlappenden imaginären Firmen gleich hoch. Sie ist insofern ein Charakteristikum der Gesamtwirtschaft.

Und nun können wir folgendes feststellen: Der oben erscheinende Ausdruck $\frac{\partial T(r ; \theta)}{\partial r}$ ist bestimmt durch die Differenz zwischen der intertemporalen Varianz der Arbeitsinputs und der intertemporalen Varianz der Konsumgüteroutputs der imaginären Firma. In den Modellen, die in der Kapitaltheorie verwendet werden (zB auch in denen, die im Schefold-Artikel behandelt werden) ergibt sich, dass diese Differenz positiv ist: das heißt, dass die intertemporale Varianz der Arbeitsinputs größer ist als die intertemporale Varianz der Konsumgüteroutputs. Es gilt dann $\frac{\partial T(r ; \theta)}{\partial r}>0$. Vgl. hierzu Weizsäcker (1971, S. 71 f), dort allerdings noch ohne Rückbezug auf die Arbeitsteilung, den ich nunmehr kurz skizziere.

Die Denkschemata der Kapitaltheorie führen somit zu dem Ergebnis, dass die Produktionsperiode für eine vorgegebene Produktionstechnik $\theta$ umso höher ist, je höher der Zinssatz ist, mit dem die Gegenwartswerte berechnet werden. Weshalb aber sollte grundsätzlich die intertemporale Dispersion der Arbeitsinputs größer sein als die intertemporale Dispersion der Outputs? Meines Erachtens steht dahinter das die Moderne charakterisierende Prinzip der Arbeitsteilung. Ich führe das an dieser Stelle nicht aus, werde den Zusammenhang zwischen Kapitaltheorie und Arbeitsteilung aber in dem in Arbeit befindlichen Text genauer darstellen. Hierzu bedarf es unter anderem einer Axiomatik für eine Theorie der Arbeitsteilung, die bisher von der Volkswirtschaftslehre nicht entwickelt worden ist. Ich unterscheide in diesem Zusammenhang zwischen einer horizontalen Arbeitsteilung und einer vertikalen Arbeitsteilung. Die horizontale Arbeitsteilung manifestiert sich in der großen Anzahl zeitlich parallel zueinander laufenden Aktivitäten, die jeweils unterschiedliches Know-How enthalten. Die vertikale Arbeitsteilung ist in Nachfolge der Mengerschen Güterordnung die intertemporale Differenzierung des Gesamtarbeitsprozesse, d.h. das intertemporale Aufeinander-Aufbauen der verschiedenen Arbeitsschritte. Die vertikale Arbeitsteilung ist verknüpft mit dem Bedarf an Kapital - und damit, wie oben dargestellt, mit der durchschnittlichen Produktionsperiode.

Die vertikale Arbeitsteilung erhält ein sinnvolles Maß in der intertemporalen Varianz der Arbeitsinputs der virtuellen Unternehmung, die voll integriert ist. Ich betrachte zuerst den Spezialfall eines Wicksell-Effekts von Null. Dem entspricht das Solow-Modell der Volkswirtschaft mit nur einem Gut, das je nach Verwendung Konsumgut oder Kapitalgut ist. Solow (1956). In diesem Fall erhalten wir

$$
w_{11} \equiv \frac{\partial w_{1}}{\partial r}=w\left\{T^{2}-\frac{\partial T}{\partial r}\right\}=0
$$

Man kann nun zeigen, dass in diesem Fall eine mögliche virtuelle vollintegrierte Unternehmung darin besteht, dass sie vom Zeitpunkt Null an stationär ist, vor dem Zeitpunkt Null aber nur für den Zweck des Kapitalaufbaus produziert. Es ergibt 
sich dann eine Konsumzeitreihe, die ab Null einen konstanten positiven Wert und vor dem Zeitpunkt Null einen Wert von Null aufweist. Der Arbeitsinput ist ab dem Zeitpunkt Null konstant. Vor dem Zeitpunkt Null wächst der Arbeitsinput mit der prozentualen Rate, die gleich der Kapitalproduktivität ist. Die Lohn-Zins-Kurve ist hier eine Gerade mit der Steigung $-k$, wobei $k$ die Kapitalintensität ist (ich bezeichne in diesem speziellen Fall die Produktionstechnik $\theta$ mit der ihr entsprechenden Kapitalintensität $k$ ). Es gilt also

$w(r ; k)=w(0 ; k)-k r$

Die Produktionsperiode ist dann

$$
T(r ; k)=-\frac{w_{1}}{w}=\frac{k}{w(0, k)-k r}
$$

und daher

$$
\frac{\partial T}{\partial r}=\frac{k^{2}}{w^{2}}=T^{2}
$$

Das bedeutet: Im Fall des Solow-Modells, also eines Wicksell-Effektes von Null, ist der Überschuss der Dispersion der Arbeitsinputs über die die Dispersion der Konsumgüteroutputs gerade gleich dem Quadrat der Produktionsperiode. Dem entspricht, dass wir in der Wahrscheinlichkeitstheorie für die Exponentialverteilung wissen, dass die Varianz gleich dem Quadrat des Mittelwertes ist.

Ein „,neoklassischer“ Wicksell-Effekt entspricht dann dem Fall, dass das Maß der vertikalen Arbeitsteilung geringer ist als beim Solow-Modell mit gleicher Produktionsperiode. Bei einem ,anti-neoklassischen“ Wicksell-Effekt ist die vertikale Arbeitsteilung größer als beim Solow-Modell mit gleicher Produktionsperiode.

Anders ausgedrückt: Eine relativ geringe vertikale Arbeitsteilung führt zu einer konvexen Lohn-Zins-Kurve bzw. zu einem „,neoklassischen“ Wicksell-Effekt. Eine hohe vertikale Arbeitsteilung führt zu einer konkaven Lohn-Zinskurve bzw. zu einem ,anti-neoklassischen“ Wicksell-Effekt.

Der Wicksell-Effekt ist damit ein Indikator für das Ausmaß der vertikalen Arbeitsteilung. Mit der Frage nach der Stabilität des Gleichgewichts hat er m. E. wenig oder nichts zu tun.

\section{Substitution als Resultat von Zinsänderungen}

In der Neo-Ricardianischen Literatur ist viel Aufhabens davon gemacht worden, dass es im Allgemeinen nicht möglich ist, einen monotonen Zusammenhang zwischen der Profitrate und der eingesetzten Produktionstechnik herzustellen. Die theoretische Möglichkeit eines „Reswitching“ ist seit der damaligen Debatte um diese Frage allgemein anerkannt. Zerstört dieses Ergebnis nicht auch die Grundlagen für eine Weiterentwicklung der Theorie von Böhm-Bawerk? 
Hierzu jedoch eine Hinweis auf mein Gesetz der intertemporalen Substitution. Die Produktionsperiode $T$ als als quantitativer Ausdruck des Ausmaßes der Produktionsumwege hängt natürlich nicht nur vom Zinssatz $a b$, sondern auch von der verwendeten Produktionstechnik $\theta$. Der Zinssatz $r$,,steuert“ die Wahl der Produktionstechnik $\theta$. Wir können schreiben: $\theta=\theta(r)$. Da die wir frei sind in der Wahl der „Namen“ für die einzelnen Produktionstechniken, können wir der beim jeweiligen Zinssatz $r$ eingesetzten Produktionstechnik den „Namen“ $r$ geben. Damit gilt $\theta(r)=r$.

Nun können wir uns interessierende Größen wie zum Beispiel den Lohnsatz w oder den Kapitalwert pro Arbeiter $v$ oder die Produktionsperiode $T$ als Funktion von $\theta$ unter Konstanthaltung von $r$ betrachten. Hierbei gehe ich in der Tradition der Kapitaltheorie davon aus, dass für ein vorgegebenes $r$ die Technik $\theta$ so gewählt wird, dass der Lohn maximiert wird. Sei also Theta die Menge der verfügbaren Produktionstechniken. Dann soll gelten

$$
w(r ; \theta(r)) \geq w(r ; \theta) ; \theta \in \text { Theta }
$$

Gleiches gilt dann auch für den Logarithmus des Lohnsatzes, den wir mit $\omega$ bezeichen. Daher

$\omega(r ; \theta(r)) \geq \omega(r ; \theta) ; \theta \in$ Theta

Wir haben dann

$$
\omega_{1} \equiv \frac{\partial \omega}{\partial r}=\frac{w_{1}}{w}=-T(r ; \theta)
$$

Jetzt machen wir eine Taylor Approximation zweiten Grades für $\omega$ um den beliebigen Wert $\bar{r}$ und $\bar{\theta}=\theta(\bar{r})$. Es sei definiert: $\Delta \mathrm{r} \equiv r-\bar{r}$. Unter Berücksichtigung obiger Ungleichung erhalten wir

$$
\begin{aligned}
\omega(r ; \theta(r))=\omega(\bar{r} ; \bar{\theta})+(\Delta r)\left(\omega_{1}+\omega_{2}\right) \\
+\frac{1}{2}\left\{\omega_{11}+\omega_{12}+\omega_{21}+\omega_{22}\right\}(\Delta r)^{2} \\
\geq \omega(r ; \bar{\theta})=\omega(\bar{r} ; \bar{\theta})+(\Delta r) \omega_{1}+\frac{1}{2} \omega_{11}(\Delta r)^{2}
\end{aligned}
$$

Wegen der obigen Ungleichung folgt zudem, dass $\omega_{2}=0$ und $\omega_{22} \leq 0$

Damit können wir ableiten

$$
2 \omega_{12}=\omega_{12}+\omega_{21} \geq \omega_{12}+\omega_{21}+\omega_{22} \geq 0
$$

Mithin $\omega_{12}=-\frac{\partial T}{\partial \theta} \geq 0$. Daher

$$
\frac{\partial T}{\partial \theta} \leq 0
$$


Abb. 1 Lohn-Zins-Kurve zweier Produktionstechniken
W

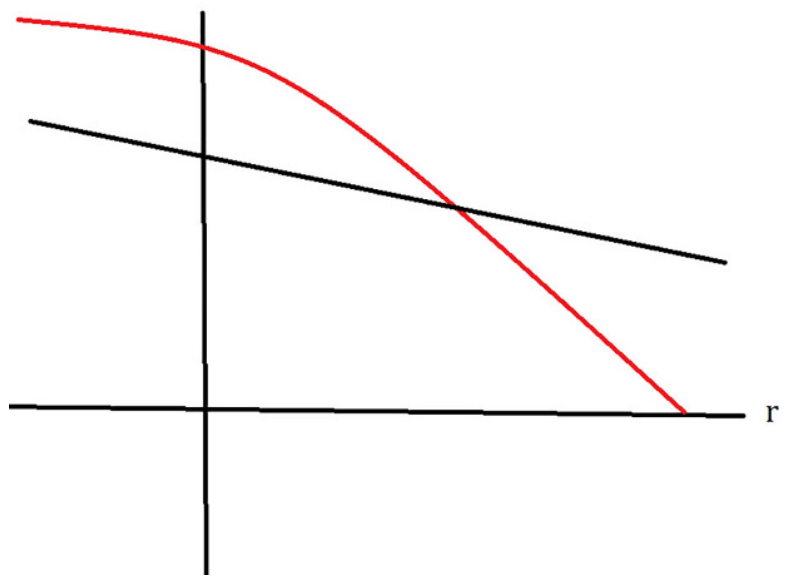

Das heißt: Mit steigendem Zinssatz verschiebt sich die Produktionstechnik derart, dass die Produktionsperiode kleiner wird, solange man das Preisgewichtungssystem zur Messung von $T$ konstant hält.

Dies aber ist ein sehr „neoklassisches“ Ergebnis: Solange wir das zinsinduzierte Gewichtungssystem (im Sinne der Gegenwartswerte) konstant halten, reagieren die physischen Größen von Arbeitsinputs und Konsumgüteroutputs auf eine Zinserhöhung derart, dass die Produktionsumwege abnehmen. Das ist das Gesetz der intertemporalen Substitution. Eine allgemeinere Variante dieses Gesetzes ist enthalten in Weizsäcker (1974).

Ihm entspricht Böhm-Bawerks Vision, dass der Zins das Steuerungsmittel der Produktionsumwege ist.

Wir können denselben Gedanken auch intuitiv-geometrisch mithilfe der SwitchPunkte zwischen zwei Techniken ausdrücken (Abb. 1).

Am Switch-Punkt ist der Absolutbetrag der Steigung der roten Lohn-Zins-Kurve höher als der der schwarzen Kurve. Mit steigendem Zins geht die Volkswirtschaft von der roten zur schwarzen Kurve über. Beim Gewichtungssystem für die Gegenwartswerte, das dem Zinssatz am Switch-Punkt entspricht, verändert sich das Produktionssystem bei steigendem Zinssatz in Richtung einer geringeren Produktionsperiode, die ja das Negative der prozentualen Steigung der jeweiligen LohnZins-Kurve ist. Das ist Böhm-Bawerk ,in a nutshell“.

\section{Literatur}

Böhm-Bawerk (1889). Kapital und Kapitalzins. Zweite Abteilung. Positive Theorie des Kapitales. Innsbruck: Wagner.

von Hayek, F. A. (1945). The Use of Knowledge in Society. American Economic Review, 35(4), 519-530 Hicks, J. R. (1939). Value and capital. London: Oxford University Press.

Smith, A. (1776). An inquiry into the nature and causes of the wealth of nations. London: W. Strahan and T. Cadell. 
Solow, R. M. (1956). A contribution to the theory of economic growth. Quarterly Journal of Economics, 70(1), 65-94.

von Weizsäcker, C.C. (1971). Steady state capital theory. Heidelberg: Springer.

von Weizsäcker, C.C. (1974). Substitution along the time axis, 27, 732-756. Kyklos.

von Weizsäcker, C. C. (2005). Hayek und Keynes: Eine Synthese. In Ordo (Bd. 56, S. 95-111). 